\title{
Expression of horseradish peroxidase in transgenic tobacco
}

A. Pellegrineschi*, M. Kist, I. Dix*, T. A. Kavanagh‡ and P. J. Dix*

*Department of Biology, St. Patrick's College, Maynooth, Co. Kildare, Ireland, tInstitute of Plant Physiology,

Biological Research Centre, Hungarian Academy of Sciences, Szeged, Hungary, and fDepartment of Genetics, Trinity College, Dublin, Ireland

\section{Introduction}

Peroxidases are common oxidoreductases present in plants, animals and micro-organisms. Plant peroxidases are classified as basic, neutral or acidic enzymes, depending on their profiles of elution during ion-exchange column chromatography or isoelectric points [1]. Horseradish (Armoracia rusticana) peroxidases (HRPs; EC 1.11.1.7), are probably the best characterized of these enzymes. They are believed to be involved in plant responses to a number of biotic and abiotic stresses, notably in the disruption of free radicals and active derivatives of oxygen. The effects of these molecules have been well described [2], and in general the presence of these compounds are 'highly destructive' for cell metabolism. However, these oxygen derivatives are involved in halogenation and hydroxylation reactions in peroxisomes and in the cell wall, suggesting that the plant cell needs a fine regulation between production and destruction of oxygen radicals. A key to the comprehension of the role of peroxidase activity in this and other stress responses could be the study of the effect of modified expression and targeting of peroxidase activity in transgenic plants. The present report describes the production of such tobacco plants using a synthetic HRP gene [3], with a range of regulatory and targeting sequences.

\section{Materials and methods}

Plant materials and tissue culture

Nicotiana tabacum I. cv. Samsun NN sterile plants were grown on MS medium [4] with $0.7 \%$ agar in a

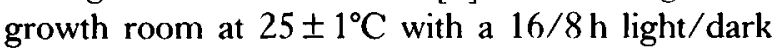
photoperiod.

\section{Construction of extensions and plasmids}

A $700 \mathrm{bp}$ DNA fragment containing sequences encoding the $\mathrm{N}$-terminal half of HRP isoenzyme $\mathrm{C}$ was amplified from total horseradish DNA by PCR using the synthetic oligonucleotides PC1 (5'TCTTTTTCTAGATATTCTATA- ${ }^{\prime}$ ) and PC2 (5'-TTACATTAACAAAGCAGTCGTG- $3^{\prime}$ ). The sequences of these oligonucleotides were based on the prxCla gene described by Fujiyama et al. [5].

Abbreviations used: HRP, horseradish peroxidase; CaMV, cauliflower mosaic virus.
The fragment was cloned into pUC18 to give pHCla. pHCl was then used as the template in a second $\mathrm{PCR}$ reaction containing the synthetic oligonucleotide primers PCN1 (5'-GATCTAAAGCTTGGATCCTTTAAAAATGCATTTC- ${ }^{\prime}$ ) and PCN2 (5'-GGTAGGGGTTAACTGAGCAGACAA- $3^{\prime}$ ) in order to amplify sequences encoding the $\mathrm{N}$-terminal amino acid extension and to place several convenient restriction enzyme sites (HindIII, BamHI, Dral and NsiI) immediately adjacent to the initiating ATG of the N-terminal extension. The resulting $120 \mathrm{bp}$ PCR product was digested with HindIII and HpaI and cloned into HindIII-Hpal-digested pUC18HRP [3] to give the plasmid pPUPN, which encodes the synthetic HRP gene with the natural $\mathrm{N}$-terminal extension.

The HRP gene constructs were excised from the intermediate plasmids pPUP, PUPC (HRP with C-terminal coding sequences), pPUPN (HRP with $\mathrm{N}$-terminal coding sequences) and pPUPNC (HRP with $\mathrm{N}$-and $\mathrm{C}$-terminal sequences) and placed under the control of the constitutive cauliflower mosaic virus (CaMV) $35 \mathrm{~S}$ promoter, a tobacco light-inducible ribulose 1,5-bisphosphate carboxylase-small subunit (RUBISCO-SSU) promoter [6], or the soybean heat shock promoter [7]. The constructs were finally introduced into Agrobacterium binary vector pBin 19 for tobacco transformation. All cloning manipulations were by standard methods, essentially as described in [8]

\section{Plant transformations and growth of plant material}

For stable transformation, the constructs were transferred into Agrobacterium tumefaciens (strain LBA 4404) as described previously [9] and introduced into the tobacco genome using a leaf disc transformation procedure [10]. The regenerated tobacco shoots were selected by growth on a medium containing MS salts and vitamins [4], 3\% sucrose and kanamycin sulphate $(100 \mathrm{mg} / \mathrm{l})$. After rooting the plants were potted and transferred to a growth room at $23 \pm 1^{\circ} \mathrm{C}$.

\section{Extraction of peroxidase and assay of enzyme activity}

Total protein was extracted from $1 \mathrm{~g}$ of leaf tissue with saline phosphate buffer (sodium acetate and 
potassium acetate), $\mathrm{pH}$ 6.2. The homogenate was centrifuged (Sorval SC285) for $10 \mathrm{~min}$ at $10466 \mathrm{~g}$, and the supernatant was retained.

The peroxidase activity was measured coloriFor the spectrophotometric assay, $2 \mathrm{ml}$ of reaction mixture was mixed with $100 \mu \mathrm{l}$ of the crude extract and the absorption was immediately measured for $30 \mathrm{~s}$ at $420 \mathrm{~nm}$. Peroxidase activity was also visualized in situ in polyacrylamide gels. For these activity gels, the proteins were extracted with the same buffer containing $50 \%$ glycerol, $1 \%$ Bromophenol Blue and $5 \mathrm{mM}$ EDTA. After centrifugation the samples were electrophoresed on an $8 \%$ polyacrylamide gel at $75 \mathrm{~V}$ for $3 \mathrm{~h}$. Subsequently the gel was incubated for $15 \mathrm{~min}$ in buffer containing $5 \%(\mathrm{w} / \mathrm{v})$ guaiacol, $0.1 \mathrm{M}$ potassium buffer $(\mathrm{pH} 6.0$ ) and $0.147 \mathrm{M}$ hydrogen peroxide.

\section{Western blot analysis}

Proteins extracted from young leaves were denaturated in SDS sample buffer, boiled for $5 \mathrm{~min}$, and resolved by $10 \%$ polyacrylamide gel electrophoresis in the presence of $0.1 \%$ SDS. Following electrophoresis, proteins were electrotransferred to nitrocellulose. The membrane was incubated in blocking buffer $(5 \%$ milk powder in distilled water) for $30 \mathrm{~min}$ and then incubated for $2 \mathrm{~h}$ with anti-HRP antibody (Dako or Sigma). After a short wash with blocking buffer the blots were incubated for $1 \mathrm{~h}$ with alkaline phosphatase secondary antibody (Sigma) and finally stained with NitroBlue Tetrazolium and bromochloroindoyl phosphate substrates (Sigma).

\section{Plant nucleic acid analysis}

DNA was extracted from plantlets regenerated in the presence of $100 \mathrm{mg} / \mathrm{l}$ kanamycin sulphate [11]. The PCR analyses were carried out using NPTIIspecific primers following the method described in [8].

\section{Progeny analysis}

The seeds from the $\mathrm{T}_{0}$ plants were surface-sterilized and germinated on MS medium with $100 \mathrm{mg} / \mathrm{I}$ kanamycin sulphate for 20 days in order to check the segregation of the NPTII gene (marker gene) in the progeny.

\section{Results}

\section{Transformation of tobacco}

The HRP gene constructs were transferred into Nicotiana tabacum cv. Samsun NN, using the

plasmid Bin 19 [9]. Five independent transformants were produced for each construct and transferred to soil. PCR analysis confirmed the presence of the NPTII gene in all of these plants. Western blot analyses revealed the presence of the HRP protein in all these plants with higher levels in those containing the gene with $\mathrm{N}$-terminal or $\mathrm{N}$ - plus $\mathrm{C}$-terminal extensions (Figure 1). Expression in the plants with the heat-shock constructs was restricted to plants induced at $40^{\circ} \mathrm{C}$.

\section{Characterization of tobacco $T_{1}$}

Seeds were produced from all transgenic plants. A 3:1 segregation ratio was found in $T_{1}$ seedlings checked for kanamycin resistance. Expression, as assessed by Western blots and enzyme assay of the ROPN and ROPNC genes (HPR with N-terminal or both extensions) under control of the CaMV promoter, was observed in all organs analysed (leaves, stem and roots) of the transgenic plants. Plants with the ROP and ROPC constructs (HPR without or with C-terminal extension) also showed expression of the recombinant proteins, but at a lower level.

\section{Peroxidase activity}

All transgenic plants showed an increase in peroxidase activity with respect to the control (Figure 2). The level of peroxidase activity, however, differed markedly depending on the terminal extensions, being lowest for the construct with the $\mathrm{C}$-terminal

\section{Figure I}

Western blot analysis showing the presence of HRP in tobacco (arrow) in constructs with the CaMV $35 \mathrm{~S}$ promoter

Lanes from left to right: $R$, seedlings of ROPNC (two lanes): plants transformed with ROPN, ROPNC, ROP and ROPC; and control.

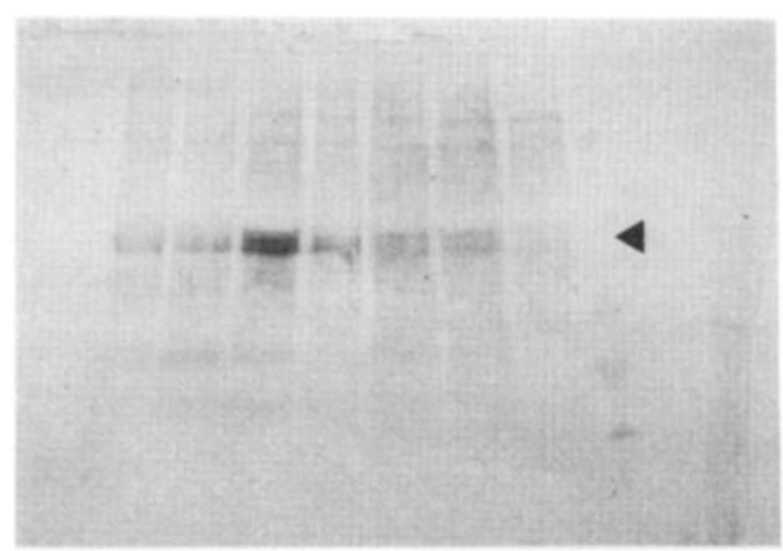


Figure 2

Peroxidase activity of extracts from leaves of transgenic and control tobacco plants

Values are absorbance values of $420 \mathrm{~nm}$ over $30 \mathrm{~s}$ from the commencement of the reaction.

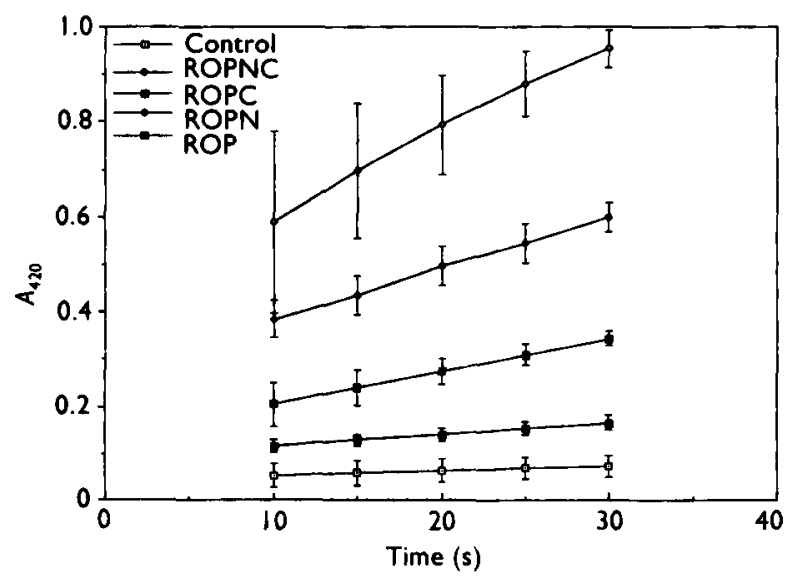

Figure 3

Gel of peroxidase activity from plants containing constructs with the CaMV $35 \mathrm{~S}$ promoter

Lanes from left to right: molecular mass marker, control tobacco plant, ROP, ROPC, ROPN, ROPNC. The arrow indicates a molecular mass of $45 \mathrm{kDa}$.

extension alone and highest for the construct with the $\mathrm{N}$-terminal extension. Activity gels confirmed the elevated levels of peroxidase, compared with control tobacco plants, and also revealed mobility differences between peroxidase bands in plants containing the HPR gene with or without the Ntermini extension (Figure 3).

\section{Discussion}

The increases in peroxidase activity in the crude protein extracts from transgenic plants supports the view that substantial overexpression can be accomplished in this way, thus providing a valuable tool for assessing the role of peroxidases in plant developmental processes and stress responses. The data also suggest that the extensions at the $C$ - and $\mathrm{N}$-termini may have a role in the control of enzyme activity, as well as in targeting. The Western blot results show the presence of HRP in the transformed plants. However, there are clear quantitative differences, with more recombinant protein in plants containing the constructs with the N-terminal extension. These differences are mirrored in the enzyme assays and activity gels, which clearly show a reduced activity when the $\mathrm{C}$-terminal extension is present. The possibility that $\mathrm{C}$-terminal targeting might lead to a reduction in enzyme activity, or influence protein secretion [12], is the subject of ongoing investigations. The activity gels clearly show an additional band of lower molecular mass when this extension is present. The role of the N-terminal extension is also under scrutiny. The transgenic material produced in this study provides a resource for the detailed dissection of the roles of the $\mathrm{C}$ - and N-terminal extensions. Localization of the recombinant enzyme will be an important part of this investigation, and also impinges on subsequent studies on peroxidases in plant development or stress responses.

We acknowledge the support of Forbairt and the EC Human Capital and Mobility Programme, which respectively allowed $\mathrm{M}$. Kis and A. Pellegrineschi to participate in this research.

1 Welinder, K. G. (1992) Curr. Opin. Struct. Biol. 2 , 388-393

2 Foyer, C. H., Descourvières, P. and Kunert, K. (1994) Plant Cell Environ. 17, 507-523

3 Smith, A. T., Sanders, S. A., Sampson, C., Bray, R. C., Thorneley, R. N. F. and Burke, J. F. (1990) J. Biol. Chem. 265, 13335-13343

4 Murashige, T. and Skoog, F. (1962) Physiol. Plant. 15, 473-497

5 Fujiyama, K., Takemura, H., Shinmyo, A., Okada, H. and Takano, M. (1990) Gene 89, 163-169

6 Mazur, B. J. and Chui, C.-F. (1985) Nucleic Acids Res. 7, 2373-2387

7 Baumann, G., Raschke, E., Bevan, M. and Schoffl, F. (1987) EMBO J. 6, 1161-1166

8 Sambrook, J., Fritsch, E. F. and Maniatis, T. (1989) Molecular Cloning: A Laboratory Manual, 2nd edn., 
Cold Spring Harbor Laboratory Press, Cold Spring Harbor

9 Bevan, M. (1984) Nucleic Acids Res. 12, 8711-8721

10 Horsh, R., Fry, J., Hoffman, N., Eichholtz, D., Rogers, S. and Fraley, R. (1985) Science 227, 1229-1231
11 Dellaporta, S., Wood, J. and Hicks, J. (1983) Plant
Mol. Biol. Rep. 1, 19-21

12 Munro, S. and Pelham, H. R. B. (1987) Cell 48, 899-907

Received 11 November 1994

\section{Homology modelling of horseradish peroxidase coupled to two-dimensional NMR spectral assignments \\ G. H. Loew*, P. Du* and A. T. Smith $\dagger$ \\ *Molecular Research Institute, 845 Page Mill Road, Palo Alto, CA 94304, U.S.A., and †Biochemistry Laboratory, University of Sussex, Brighton, Sussex BNI 9QG, U.K.}

\section{Introduction}

Horseradish peroxidase (HRP) is a member of the plant peroxidase superfamily [1]. It is able to utilize hydrogen peroxide to catalyse the one-electron oxidation of a wide range of aromatic phenols and anilines. It has been proposed [2] that such aromatic hydrogen donor substrates, as well as phenylhydrazine [3], bind to peripheral sites near the $\delta$-meso and $8-\mathrm{CH}_{3}$ haem edge about 8-11 $\AA$ from the iron, while other substrates, typified by benzhydroxamic acid, bind to a distal haem site. Although crystallographic work is under way [4], no high-resolution structure is currently available. NMR studies of the paramagnetic cyano HRP complex containing wildtype and HRP protein variants have proven particularly powerful [5-7] in providing information about residues in proposed peripheral sites as well as key residues near the distal and proximal sides of the haem. On the distal side, the highly conserved residues Arg-38, His-42, Phe-41 and Phe-152 have been identified [5-8]. On the proximal side, His170 was identified early as the haem-iron ligand [7] and, in more recent work [9], observed cross-peaks with the $4 \mathrm{H} \beta$ vinyl group of the haem were assigned to the NH group of Gly-169. However, three other assignments remain in doubt: an unidentified isoleucine-X [6] originally thought to be Leu-237 that should be close to the 8-methyl group, an unidentified residue originally assigned as Tyr- 185 but subsequently questioned [10], and an unidentified aliphatic residue with two protons assigned to observed cross-peaks with the $\mathrm{H} \beta_{1}$ and $\mathrm{H} \beta_{2}$ protons of the proximal His-170 residue [9].

Abbreviations used: HRP, horseradish peroxidase; 3D and $2 \mathrm{D}$, three- and two-dimensional respectively; $\mathrm{CCP}$, cytochrome $c$ peroxidase; ARP, Arthromyces ramosus peroxidase; LiP, ligninase; SCR, structurally conserved region; MD simulations, molecular dynamic simulations.
While NMR studies of the paramagnetic HRP-CN complexes have provided useful information about the position of local residues near the paramagnetic haem unit, these techniques cannot, by themselves provide a global three-dimensional (3D) structure of the protein. However, when NMR results are combined with the technique of homology modelling used to construct 3D models of the protein, they can contribute to the selection of the most plausible 3D model. Thus homology modelling provides the missing link between local and global structural information that can be derived from NMR studies, and thus enhances its usefulness.

In the work reported here, we have used the known crystal structures of three members of the superfamily, cytochrome $c$ peroxidase (CCP) [11], Arthromyces ramosus peroxidase (ARP) [12] and ligninase (LiP) [13] as templates to construct $3 \mathrm{D}$ models of HRP. By comparing several candidate models obtained using the distances between specific substituents of the haem and nearby residues deduced from NMR studies, the most plausible model could be selected and the unknown residues in the peripheral binding site identified.

\section{Methods}

In this section we briefly summarize the steps used to: (1) construct the initial 3D models of HRP; (2) refine the initial models; (3) construct and refine their corresponding cyano complexes; and (4) further refine the most plausible model. Details of these models are described elsewhere [11].

\section{Construction of initial full protein models of HRP C}

The structures of three members of the plant peroxidase superfamily [1] to be used as templates, CCP [12], LiP [13] and ARP [14] were obtained 